\title{
Urgences
}

\section{La Renaissance à Gray}

\section{Pierre Ouellet}

Numéro 27, mars 1990

Images imaginaires

URI : https://id.erudit.org/iderudit/025579ar

DOI : https://doi.org/10.7202/025579ar

Aller au sommaire du numéro

Éditeur(s)

Urgences

ISSN

0226-9554 (imprimé)

1927-3924 (numérique)

Découvrir la revue

Citer cet article

Ouellet, P. (1990). La Renaissance à Gray. Urgences, (27), 67-70.

https://doi.org/10.7202/025579ar d'utilisation que vous pouvez consulter en ligne.

https://apropos.erudit.org/fr/usagers/politique-dutilisation/ 


\section{La Renaissance à Gray Pierre Ouellet}

Mesme la froide mort qui si fort nous estonne Ne ravit point la vie, ains seulement nous donne Tant soit peu de respit pour le temps avenir. J.-B. C.

Arc-lès-Gray, $1^{\text {er }}$ janvier 1990

On ne sait pas, et ne le saura jamais, à quel âge il mourut, tout près d'ici, vers l'an 1635 . Chose certaine: c'est en six mois à peine, dans la jeune vingtaine, qu'il écrivit sans discontinuer les 434 sonnets qu'on lui connaît - chaque poème tout entier tourné, comme un jeune visage vers le nom vieillissant qui, bientôt, sur le tombeau, continuera de le nommer en vain, vers le bout, dit-il, du chemin de sa vie, ou à mieux dire du glissoire de la fluante mortalité.

Chaque poème tourné comme un regard: sur soi, où c'est un autre que l'on découvre: qui porte le nom qu'on a reçu comme le deuil, désormais, d'un visage défait, que l'on ne reconnaît plus. Il a, plutôt que composé des vers, décomposé les traits, un à un, de la mort qui chaque jour un peu plus nette se dessine sur notre face, qu'elle décolore, efface: Lors le rit se termine en complainte et douleur Florissant au matin comme une belle fleur Qui le soir approchant se ternit et s'efface (sonnet no CCLXV).

J'aime qu'on ne soit pas certain du jour de sa naissance, quand la date de sa mort est à peu près sûre, comme son penchant, dès sa prime jeunesse, pour le glissoire de la fluante mortalité - c'est comme s'il n'était pas encore né, lui, qui n'a fait que mourir. Il s'est en quelque sorte inventé une naissance qui ne s'est jamais réalisée. Quand il publie pour la première fois, en 1594, il affirme que la barbe commence à peine à lui pendre au menton, alors qu'à la mort de sa mère, quelques années plus tard, il déclare avoir 35 ans - comme si naître, pour lui, n'avait pas la nécessité de la mort, sa vérité, mais, plus contingent même que vivre à chaque instant, relevait de quelque coup de dés qu'on peut à tout moment 
relancer, trichant jusqu'avec le hasard, parce que toute naissance, dès le principe, serait fausseté. Elle nous détourne de cette seule chose, qui soit évidente - parce qu'on y assistera bientôt comme jamais on ne l'aura fait: à sa venue au monde -, soit à sa propre, inéluctable, disparition, ce qu'il appelle: sa venue au vuide.

Car on viendrait à cela, aussi - et à grands pas. Le néant arrive, lui aussi, bien plus sûrement que le moindre fait, et nous sommes tous, l'un comme l'autre, sa seule, inévitable destination, dont rien, pas même vivre, ne le détourne, qui ne peut jamais qu'en retarder la marche, le séduisant en chemin (lui, il aurait dit: par le poeme, qui l'accompagne, car la parole, dit-il, emprunte les routes les plus difficiles, où elle est sûre de rencontrer le pire - ce dont seul elle peut, en ce monde-ci, être témoin, quand elle n'est pas aveugle).

Il n'a pas eu le temps de naitre, se consacrant tout entier à cette éternité: mourir. Voilà pourquoi on ne s'entendra jamais sur l'âge véritable de ce non-né, qui est sans âge et notre contemporain: il continue de mourir sous nos yeux, qui les fixons sur ses vers comme pour en hypnotiser le mouvement, en méduser la course, folle, vers la toute fin, en immortaliser la chute. Au lieu de quoi c'est notre propre regard qui est emporté - et va s'échouer: sur la clausule de quelque phrase qu'une autre aussitôt recommence, comme si la plage où notre vision fait à tout moment naufrage formait en fait, vagues sur vagues, plus hautes l'une que l'autre, une mer plus forte, et déchaînée, que le grand large sous la tempête.

Pas de terre ferme, ici, qui n'ait été secouée, dont le devenir incessant ne repose à chaque seconde sur l'écho prolongé de ce tremblement-là: premier - quand un nom fut donné aux choses, qui le reçurent comme un choc, en même temps que le jour, et qu'à chaque homme eut été fait don d'une langue et de la vie, en un même présent, qui lui permirent, par bruits et fureurs interposés, de l'absorber: ce coup mortel, le relayant dans quelque écho frémissant où il y a l'histoire, bien sûr, et sa furie, mais le poème, aussi, et ses bruissements.

Je le vois comme ça: lui - jamais remis du nom qu'on lui donna, parmi les choses inanimées, innommées, dont la muette désignation nous cache encore la vie secrète, et ne s'en remettant que développant en lui le don de la parole, reçu telle une mission, afin que par sa bouche, dit-il, jamais 
fermée sur autre chose qu'un cri retenu qui l'ouvre encore plus grande, des pans entiers du monde en dormition dans le sans-nom, le sans-visage, soient révélés à l'homme, mis en lumière - peu important, dès lors, que cette révélation s'appelle ou non la mort, que certains nomment résurrection.

Le pays qui l'a vu naître, aveugle comme tout nouveauné, et l'aura vu mourir, le regard égaré, lui fut toute sa vie le miroir qu'on tend, non pas aux yeux, déjà fermés, mais aux lèvres entrouvertes d'un mourant, afin d'y surprendre le moindre souffle de vie dans quelque buée. Cette légère brume, sur Gray, qui est comme le voile permanent d'un nom, imperceptible, sur le visage qu'il nomme, et qualifie (le nom est, croit-il, l'épithète absolue), n'avait plus été pour lui que cela: les centaines de souffles que les Graylois perdent à chaque instant, avec un peu d'âme, embuant l'air et le ciel, sans tain, qui leur revoient, comme l'écho le seul son, jamais le sens, d'un mot, d'une voix, une image toute embrouillée de leur propre terre: ce vide, en fait, que les seuls corps étendus comblent, un jour ou l'autre. C'est un pays, dit-il, où les larmes précèdent éternellement les yeux qui les pleurent. La vallée de la Saône, là, c'était l'imperceptible ride qu'avait creusée le pleur unique, et continu, que versent les paroles de l'homme sur le visage du monde d'où naît alors, pour qu'on s'y voie: plus vrai que nature, une image de soi noyée dans sa propre source: au fond des yeux, devenue fleuve.

Son regard avait rencontré dans ces paysages indéfinissables de Franche-Comté, en Haute-Saône, dont Gray était alors l'un des chefs-lieux les plus importants, qui fut même, pendant deux siècles, une vicomté, le miroir embu, comme on dit parfois d'un tableau aux couleurs ternies, où pussent apparaître enfin, disparaissant, les quelques traits d'un visage dans lesquels il se reconnût un peu, le temps d'une description. Il ne se serait pas appelé Jean-Baptiste pour rien: il vivait dans un éternel Jourdain, dont les crues escaladant le ciel retomberaient à chaque instant sur lui, comme les larmes dans lesquelles seules nous recevons notre nom, que les dieux reconnaissent.

Chaque poème rebaptisait l'univers: lui donnant un nom nouveau qui lui rouvrirait chaque fois un autre ciel. J.-B. immergeait le monde dans le langage, qui fut comme une bruine plus fine, sur chaque chose, que le grain permanent sur Gray, et qui passât aux yeux des hommes pour une légère pellicule 
70

détachée des cieux, un film de voix (qui fût la peau de l'âme, une fois le corps quitté), dont se couvrirait le réel pour souligner, bien plus que la cacher, sa nudité véritable, que lui, J.-B., la connaissant de près, appelait par son nom propre: la Viduité. Cet homme donnait le sentiment que tout concourait à disparaître, mais dans un tel ultime éclat que c'eût été cela, en fait, apparaître vraiment, quand l'être est à bout. Que le monde n'en peut plus - qui aurait tout donné, déjà, reçu du ciel qui s'est tari.

Les premières fois que je l'ai lu, c'est l'horizon visible de ma fenêtre aujourd'hui que j'aperçus: la ligne à peine brisée d'une solitude immense qui passerait partout comme elle sépare le ciel de la terre, inexorablement. Ici, à Arc-lès-Gray, dans la proximité où J.-B. vit le jour et la nuit en une même vision, si je puis dire, qui fut la frontière quatre cent trente-quatre fois franchie entre vivre et mourir, on nè- peut qu'ouvrir les yeux plus grands, toujours, sur ce monde-là, auquel chaque sonnet donna naissance comme un grand coup, fatal, en plein visage. C'est l'être défiguré de chaque chose qui apparait à l'homme, seulement, jamais l'image du fond des yeux où les mille apparences du monde se concilient la vérité, indéformable.

Ici, c'est comme la chute de chaque sonnet qui s'imposerait: un vaste plateau dont on devine, au fond des plus longues perspectives, l'arête coupante, au-delà de quoi autre chose existe, qui fut coupée du monde, et dort au creux des plus profondes reculées, qu'un cri ou un regard jetés, telle une pierre, éveilleraient net, en un écho, en une image, où l'on trouve enfin ce qui échappe à la voix nue comme à la vue, quand leur nudité ne les a pas retirées tout à fait de ce qu'elles chantent, ou voient.

L'unisson que l'on entend au bout de chaque poème, quand le vers s'arrête, brusque, dans le blanc définitf: ce clair calcaire des crêtes et des corniches où le monde se dresse en vain, devant nos yeux, que leur vision ferait baisser, toucher le sol fuyant sous nos pieds, c'est l'écho réépousant la voix pour dire, plus fort que le silence final, l'accord parfait des choses avec le nom qui nous les donne, comme le paysage s'accorde soudain au visage de l'homme, illuminé d'un même regard, qui vient d'au-delà l'horizon commun au mot, à l'être, et à la vue, dont toute clausule, toute fin de phrase, montre à jamais l'éloignement infini, par quoi J.-B., toute sa vie, aura été ébloui * - et moi avec lui, qui me suis enterré ici, à Arc-lès-Gray. 